\title{
Distribution and evaluation of sense of coherence among older immigrants before and after a health promotion intervention - results from the RCT study promoting aging migrants' capability
}

This article was published in the following Dove Press journal:

Clinical Interventions in Aging

\begin{abstract}
LA Arola ${ }^{1-5}$
E Barenfeld ${ }^{1-3}$

S Dahlin-Ivanoff ${ }^{-3, *}$

G Häggblom-Kronlöf ${ }^{1-3, *}$

'Institute of Neuroscience and Physiology, Section for Health and Rehabilitation, The Sahlgrenska Academy, University of Gothenburg, Sweden; ${ }^{2}$ Centre for Person-Centred Care (GPCC), The Sahlgrenska Academy, University of Gothenburg, Gothenburg, Sweden; ${ }^{3}$ Centre for Ageing and Health - Agecap, The Sahlgrenska Academy, University of Gothenburg, Gothenburg, Sweden; ${ }^{4}$ National Graduate School on Ageing and Health - SWEAH, Faculty of Medicine, Lund University, Lund, Sweden; ${ }^{5}$ Department of Health and Welfare, Arcada University of Applied Science, Helsinki, Finland

*These authors contributed equally to this work
\end{abstract}

Background: The migration process can be a threat to a person's sense of coherence (SOC) and to their ability to experience life as comprehensible, manageable, and meaningful. Seen from a salutogenic perspective, this may have a negative impact on the experience of health.

Purpose: We describe the distribution of SOC and its components among older persons with an immigrant background now aging in Sweden. In addition, we evaluated whether a groupbased health promotion program with a person-centered approach could support the SOC among older persons in this group.

Materials and methods: A randomized controlled trial with postintervention follow-ups at 6 and 12 months was conducted with 131 independently living persons aged $\geq 70$ years from Finland and the Balkan Peninsula. Participants were randomly allocated to an intervention group (4 weeks of group intervention and one follow-up home visit) and a control group (no intervention but access to ordinary health care services). The outcome measure was the SOC measured by SOC-13. Chi-square and ORs were calculated.

Results: There was a significant improvement in total SOC scores for the intervention group at 6-month follow-up. Also, the ORs for the SOC components were higher in the person-centered intervention group. However, we found no significant between-group differences nor did the effect last until the 12-month follow-up.

Conclusion: Persons who have lived a long time in a host country after migration seem to have a SOC similar to native-born persons. Interventions with a person-centered approach could support the SOC by capturing individual life situations. Such interventions could support older persons by making everyday life more comprehensible and manageable and helping them to cope with challenges in daily life caused by aging.

Keywords: sense of coherence, salutogenesis, health, old age, migration

\section{Introduction}

The point of departure for this article is the health situations among aging labor immigrants. During the middle of the last century, large numbers of people migrated to countries in North Europe as labor immigrants. It was widely assumed that they would return to their country of origin after a while, but this did not happen. Instead, these people chose to stay permanently in the host country and now constitute a subgroup of older individuals who are aging in a country which is not their country of birth. ${ }^{1}$

According to the United Nations (UN), over $12 \%$ of the population worldwide is older than 60 years. $^{2}$ At the same time, approximately $5 \%$ of the total worldwide
Correspondence: LA Arola Arcada UAS, Jan-Magnus Janssons plats I, 00560 Helsinki, Finland

Tel +358207699422

Email annikki.arola@arcada.fi (c) (1) (2) 2018 Arola et al. This work is published and licensed by Dove Medical Press Limited. The full terms of this license are available at https://www.dovepress.com/terms.php

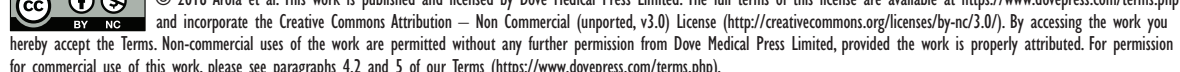


population of older people are migrants. ${ }^{3}$ In several countries, there are large population groups who migrated in their youth and are now aging in the receiving country. ${ }^{1,4}$ In Sweden, the majority of those born abroad comes from the Nordic countries (39\%) and from the Balkan Peninsula $(9 \%) .{ }^{5}$ Because the group of older immigrants have become a growing group in society, their health and living conditions cannot be ignored. ${ }^{6,7}$

Maintaining health in old age is generally both a challenge and a goal for individuals and for society. Research has shown that older people of immigrant origin are frail due to poorer health status than their native-born peers. ${ }^{8,9}$ Frailty and the immigration process reportedly represent a double burden for older immigrants, ${ }^{10}$ which jeopardizes the experience of health. The group of older immigrants has also been described as a homogeneous group with specific needs. ${ }^{6}$ However, these descriptions may risk maintaining a stereotype picture of older immigrants as passive victims of their background..$^{11}$ Additionally, earlier research has adopted a pathogenic perspective on health ${ }^{12}$ without recognizing the resources people themselves may have and which they use to shape their daily lives so as to experience health despite frailty. ${ }^{13}$

Failure to recognize this group of older persons and their experience of health is tantamount to a form of inequality regarding health and the opportunity to receive support to maintain health in daily life. Because health can be seen as a fundamental factor that influences wellbeing, ${ }^{14}$ there is a need for research focusing on exploring the experience of health among older persons aging in migration. Additionally, there is a need to explore how to support factors that contribute to the experience of health in everyday life.

This article takes a salutogenic approach ${ }^{12}$ to describe health and health resources among older persons aging in migration. The salutogenic perspective on health emphasizes the importance of a sense of coherence (SOC) in everyday life. SOC refers to the capacity to act and respond to stressful situations in life and has been strongly correlated with various health-related outcomes. SOC is high when daily life is experienced as meaningful, when daily events are comprehensible, and when persons have necessary resources to manage challenges in daily life. Meaning in daily activities is embedded in culture via specific signs and symbols. The specific signs and symbols consist of patterns, beliefs, and behaviors in our surrounding, which gives the perception of what the "appropriate" way of functioning is in specific situations. ${ }^{15}$ In an unfamiliar or new context, the embedded meaning in activities may be hidden. If the embedded meaning in everyday life is weak, persons may experience a lowered sense of comprehensibility and manageability in daily life. Thus, when the cultural context changes, for example in connection to migration, the possibilities to experience daily life as comprehensible and manageable may be affected, thus decreasing SOC. ${ }^{16}$ Persons who have migrated from one culture to another might experience fewer opportunities to engage in meaningful activities, ${ }^{17,18}$ which can be compounded by challenges interpreting the signs and symbols embedded in a new cultural context. Difficulties interpreting and understanding the new context may include difficulties in understanding the health care system, thus complicating access to health care services. ${ }^{19}$

According to Slootjes et al, ${ }^{16}$ the migration process can have an impact also on the three components of SOC. Comprehensibility can be influenced by differences in sociocultural norms that make it difficult to understand external expectations from the environment. Additionally, there may be inconsistencies in expectations from native-born persons vs compatriots. Furthermore, being a member of a minority group in a host country may negatively influence manageability due to restricted opportunities to use the resources a person has. This could include imbalances in which an individual has personal resources (such as education) that they are unable to use (underload) or imbalances in which the pressure from the environment is too high, challenging the individual excessively (overload). If both comprehensibility and manageability are negatively influenced, it may be difficult to find meaning in daily life. This can affect one's motivation to act and meet daily challenges..$^{20}$ Meaningfulness can also be affected in a migration context when the person is not included in decisions connected to important and meaningful social situations ${ }^{16}$ and decisions regarding daily life. This may create a sense of being objectified with no say in certain matters. ${ }^{12}$ This scenario may occur, for example, if the person's language skills are not sufficient to enable communication with health care representatives about needs and wishes in daily life and health-related factors. ${ }^{21}$ Ultimately, the effect of migration on the everyday life and experience of health of a person depends on 1) the person's ability to cope with demands and stressors connected to daily life, ${ }^{1} 2$ ) opportunities to manage daily life in a new context, ${ }^{8}$ and 3) opportunities to engage in meaningful activities. ${ }^{22,23}$

To be able to experience health when aging in migration constitutes the possibility to experience one's everyday 
life as meaningful, comprehensible, and manageable. SOC is hereby a critical resource that enables the person to face challenges, ${ }^{12}$ such as challenges caused by migration. Even if SOC seems to be rather stable over time, especially in adulthood, Antonovsky and Elfstadius did not see SOC as immutable without possibilities for small changes depending on the situation. Antonovsky and Elfstadius ${ }^{12}$ suggested that planned and structured interventions for the promotion of health could be a way to temporarily influence SOC and thereby support health. However, according to Antonovsky and Elfstadius, this can be done by creating interventions with a strong emphasis on encounters between the person and professionals, with the aim of supporting the person to create an understanding of the challenges faced in daily life. Thus, a health promotion intervention with a person-centered approach $^{24}$ may be a powerful way to support a person to find resources to manage such challenges, which threatens health. This may increase the possibility that individuals will experience everyday life as comprehensible, manageable, and meaningful. ${ }^{12}$

Previous cross-sectional studies have described the distribution of SOC among native-born persons ${ }^{25,26}$ and persons who have undergone migration. ${ }^{27}$ Fewer studies have explored the distribution of SOC over time or focused on older labor immigrants and the distribution of SOC among these older persons. Additionally, studies, which evaluate the effect of health promotion on SOC, are scarce. Thus, the aim of this study was to describe the distribution of SOC and its components among older labor immigrants, now aging in migration, and to evaluate whether a health promotion intervention with a person-centered approach could influence SOC 6- and 12-month postintervention.

\section{Materials and methods Trial design}

We conducted a randomized controlled trial (RCT) that had a cross-sectional and a longitudinal design. The study was part of the Promoting Aging Migrants' Capability project (PAMC). ${ }^{34}$ The PAMC was a two-armed RCT with parallel assignment in an intervention group and a control group. The trial addressed independent living older persons who had migrated from Finland and the Balkan Peninsula to Sweden because these groups are the two largest groups of immigrants in Sweden. The regional Ethical Review Board approved the study (Reg nr: 821-11). Regional Ethical Review Board, PO Box 401, SE405 30 Gothenburg. The participants gave written informed consent. The trial is registered at ClinicalTrials. gov (NCT01841853).

\section{Participants and settings}

The recruitment process was conducted in three steps to recruit the target number of participants. First, we used an official register to randomly select participants from a suburban area of a middle-sized town in western Sweden. Second, the same procedure was used in another suburban area with similar demographics. In the third step, we conducted snowball sampling ${ }^{30}$ in which participants from the middle-sized town were actively recruited. Additional information about the recruitment process is reported in the study protocol..$^{34}$ The demographics of the two suburban areas and the middle-sized town are presented elsewhere. ${ }^{16}$ Inclusion criteria for participation was as follows: Persons who had migrated from Finland or from four countries in the Balkan Peninsula (Bosnia-Herzegovina, Croatia, Montenegro, and Serbia) living in ordinary housing in a suburban district, aged 70 years or older, and independent in terms of the activities of daily living as measured by the activities of daily livingstaircase. ${ }^{28}$ Persons with impaired cognitive function, as measured with the Mini-Mental State Examination (MMSE), were excluded. ${ }^{29}$ Specifically, individuals who scored below $80 \%$ on administered items from the MMSE at baseline were excluded and referred to appropriate health care services. In total, 131 persons fulfilled the inclusion criteria and consented to participate. Of these, 88 participants were allocated in the first step, 37 participants in the second step, and six participants were recruited via snowball sampling. The study protocol contains details regarding the study setting and the Swedish health care system. ${ }^{34}$

\section{Procedure}

In the first and second steps, letters were sent to persons randomly selected from the official register. The letter described the study design, protocol, and expectations associated with participation. Potential participants were also informed that participation was voluntary and that they would be able to withdraw from the study at any time, without giving any reason for their withdrawal. One to 2 weeks after the letters were sent, eligible persons were telephoned. If eligible participants did not supply a phone number, a new letter was sent in which the person was asked to contact the research leader. Snowball sampling ${ }^{30}$ was conducted by asking former participants and members of the reference group to advertise the study to potential participants. The program was also advertised on a local radio program. Prospective participants were asked to contact the research leader for further information, at which point trained research assistants assessed whether they met the inclusion criteria. The assessments and distribution 
of study information were conducted in the participants' preferred language or in both Swedish and their preferred language. The intervention was implemented in two suburban areas located close to the recruited population. A major proportion of the inhabitants of the neighborhoods in which recruitment and intervention took place were born abroad. These neighborhoods had a lower average income and educational level compared with the overall level in the district. The details of the study setting have been reported in the study protocol ${ }^{34}$ (see Table 1 for further information about the demographic characteristics of the participants).

\section{Interventions}

Senior group meetings with one follow-up home visit The intervention consisted of four group meetings, named "senior meetings" (SMs) and one follow-up home visit. The interventions were conducting using a person-centered approach. Person-centered approach aims to create encounters where participants support one another to make decisions in daily life that support their overall health. ${ }^{24}$ The starting point is a focus on the person as an individual person beyond any diagnoses or illnesses. ${ }^{31}$ This approach enables participants to share knowledge and strategies with one another. Additionally, it encourages participants to explore their experiences in attempting to maintain and strengthen their health in daily life, together with other participants and with professionals. This creates better opportunities for the person to transfer knowledge into action in daily life. ${ }^{32}$ This form of peer learning within a group supports the awareness of health resources in daily life among participants. ${ }^{33}$ In the SMs, the focus of the meeting was directed by the experiences of the participants, such that the themes varied according to participants' preferences and needs. A group of professionals including an occupational therapist, physiotherapist, registered nurse, and social worker organized the SMs, and each professional was responsible for one meeting. A written booklet in the participants' native language supplied written information about health-related issues. Participants were encouraged to read the written information beforehand so that they had the opportunity to reflect on the various issues from a personal perspective. The study protocol contains detailed information about the content of the booklet and the roles of the professionals. ${ }^{34}$ According to the preference of each participant, SMs were conducted in Swedish, the participant's native language, or a combination of both languages. The program had access to an interpreter if the participants wished to use one. A team leader attended all meetings with the aim of creating continuity in the meetings and stimulating the group process.

Table I Demographic characteristics of participants at baseline

\begin{tabular}{|c|c|c|c|}
\hline & $\begin{array}{l}\text { Control } \\
n=75(\%)\end{array}$ & $\begin{array}{l}\text { Intervention } \\
\mathrm{n}=56(\%)\end{array}$ & $\begin{array}{l}\text { Total } \\
\mathrm{n}=131 \text { (\%) }\end{array}$ \\
\hline $\begin{array}{l}\text { Age range } \\
\text { (mean, SD) }\end{array}$ & $\begin{array}{l}70-84 \\
(74.2,3.4)\end{array}$ & $\begin{array}{l}70-82 \\
(74.0,3.4)\end{array}$ & $\begin{array}{l}70-84 \\
(74.1,3.4)\end{array}$ \\
\hline Male, n (\%) & $29(52)$ & 37 (49) & $66(50)$ \\
\hline Living alone, n (\%) & $32(43)$ & $3 I(5 I)$ & $63(48)$ \\
\hline $\begin{array}{l}\text { Type of housing } \\
\text { Tenant } \\
\text { Owner of apartment } \\
\text { Owner of house } \\
\text { Others } \\
\end{array}$ & $\begin{array}{l}38(5 \mathrm{I}) \\
17(23) \\
19(25) \\
1(1)\end{array}$ & $\begin{array}{l}30(54) \\
9(16) \\
16(28) \\
1(2)\end{array}$ & $\begin{array}{l}68(52) \\
26(20) \\
35(27) \\
2(1.5)\end{array}$ \\
\hline $\begin{array}{l}\text { Education, } \mathrm{n}(\%) \\
\text { Tertiary education } \\
\text { Low education }^{\mathrm{b}}\end{array}$ & $\begin{array}{l}12(16) \\
13(18)\end{array}$ & $\begin{array}{l}8(14) \\
15(27)\end{array}$ & $\begin{array}{l}20(16) \\
28(22)\end{array}$ \\
\hline $\begin{array}{l}\text { Migrated from, n (\%) } \\
\text { Balkan Peninsula } \\
\text { Finland }\end{array}$ & $\begin{array}{l}38(5 \mathrm{I}) \\
37(49)\end{array}$ & $\begin{array}{l}22(39) \\
34(61)\end{array}$ & $\begin{array}{l}60(46) \\
71(54)\end{array}$ \\
\hline $\begin{array}{l}\text { Years lived in Sweden, } \mathrm{n}(\%) \\
\quad \geq 2 \mathrm{I} \text { years }\end{array}$ & $63(84)$ & $5 I(91)$ & II4 (87) \\
\hline $\begin{array}{l}\text { Reason for migration, n (\%) } \\
\text { Labor } \\
\text { Refugee } \\
\text { Family } \\
\text { Others }\end{array}$ & $\begin{array}{l}20(27) \\
17(23) \\
9(12) \\
27(37)\end{array}$ & $\begin{array}{l}27(50) \\
9(17) \\
7(13) \\
11(20)\end{array}$ & $\begin{array}{l}47(37) \\
26(20) \\
16(13) \\
38(30)\end{array}$ \\
\hline Good self-rated ability to speak Swedish in contact with authorities, $n(\%)$ & $66(88)$ & $5 I(9 I)$ & II7 (89) \\
\hline
\end{tabular}

Note: aniversity $\geq 3$ years among people aged $65-74$ years; bfor people aged $\geq 65$ years. 
Two weeks after the last SM, the participants were given the option to schedule follow-up home visit. These follow-up visits would give the participants the opportunity to pose any individual questions that had occurred after the last meeting. One of the professionals who had been part of the SM program made the visits.

The SMs were implemented according to the study protocol. ${ }^{34}$ Participation in at least two SMs by more than half of the participants randomized to the health promotion intervention was deemed a reasonable adherence rate. ${ }^{35} \mathrm{~A}$ total of $57 \%(\mathrm{n}=32)$ of the participants attended all four SMs, while $16 \%(n=9)$ attended three, $9 \%(n=5)$ attended two, and $11 \%(\mathrm{n}=6)$ attended one SM. Four participants did not attend any SMs. Thus, $82 \%$ of the participants achieved the feasible adherence rate.

\section{Control group}

Participants in the control had access to ordinary range of health care services, provided by the urban district. The aim of the service was to support older persons to be able to live as independently as possible. This included various services, supporting independent living, eg, meals on wheels, help with shopping and cleaning, help with personal care and a transportation service. Additionally, at the baseline and follow-up assessments, participants were given information about where to access health care if they required it urgently.

\section{Outcomes}

Data were collected from 2012 to 2016. Members of the interdisciplinary team or trained research assistants conducted the baseline assessments according to the guidelines for the different outcome measures. ${ }^{34}$ Research assistants conducted the follow-up assessments at 6 months and 12 months.

\section{SOC}

We used the 13-item Orientation to Life Questionnaire to assess SOC. ${ }^{12}$ The SOC questionnaire had three interrelated components: five items connected to comprehensibility (eg, "do you have the feeling that you are in an unfamiliar situation and don't know what to do?"), four items connected to manageability (eg, "how often do you have feelings that you're not sure you can keep under control?"), and four items connected to meaningfulness (eg, "how often do you have the feeling that there's little meaning in the things you do in your daily life?"). Scoring was based on the Likert scale, and response options ranged from (1) "very seldom or never" to (7) "very often."
As per the SOC-13 scoring guidelines, the scores for five items in the SOC-13 were reversed so that a high score on each item indicated a high level of meaningfulness, comprehensibility, and manageability. Maximum scores for the different components are as follows: 35 scores for comprehensibility, 28 scores for manageability, and 28 scores for meaningfulness. The total scores ranged from 13 to 91 and a high score indicated strong SOC. The SOC instrument is a reliable, valid, and feasible instrument. Additionally, the instrument is also cross-culturally applicable. SOC-13 scores have been found to be stable over time (test-retest correlations at a 1-year follow-up ranged from 0.69 to 0.78$).{ }^{36}$

\section{Sample size, random allocation, and blinding}

We performed a power calculation based on one of the outcome measures in the preceding study "Elderly Persons in the Risk Zone," ${ }^{37}$ using the Berg balance scale as a basis. We found that a significance level of $\alpha=0.05$ was needed to reach a power of $80 \%$, and each study arm required a sample of 65 participants to detect a difference of $\geq 15 \%$ between the groups. ${ }^{34}$ The random allocation was stratified to enroll an equal amount of people from Finland and from any of the selected four countries in the Balkan Peninsula: Bosnia-Herzegovina, Croatia, Montenegro, and Serbia. After the baseline assessment, participants were randomly assigned to the intervention group or to the control group using group assignments contained in opaque, sealed envelopes. A researcher uninvolved in the enrollment or the intervention organized the randomization, which was performed after the baseline assessments. Due to the nature of the intervention, the participants, professionals, and assessors were not blinded in this study. However, those assessing the intervention outcome were blinded from group assignment for the majority of participants.

\section{Statistical analyses}

We used descriptive and inferential statistics to compare the intervention and control groups regarding different aspects of SOC. First, we calculated the baseline distribution of SOC scores and the three SOC components, ie, comprehensibility, manageability, and meaningfulness. Second, to assess changes from the baseline to the respective follow-up, the participants were dichotomized into "maintained/improved" or "nonmaintained" SOC for the total SOC score and the different components of SOC. We used a chi-squared test to examine differences in the proportions of participants who maintained/improved their scores at the follow-up assessments. We then calculated the OR. 
In addition, we analyzed movement between different levels of SOC (low, medium, and high) from the baseline to follow-up. The scores were divided into quartiles that set the limits for low (13-63), medium (64-79), and high (80-91) SOC levels (11). The participants were dichotomized into strengthened, maintained, or deteriorated SOC, depending on how their score changed from the baseline to follow-up. We compared the movement between SOC levels, in the participants in the control group with that of those in the intervention group. We focused on the movement between the different SOC levels and not on the total scores. We used a chi-squared test to examine between-group differences in SOC levels, and ORs were calculated to see the odds for change for the participants in the control group and intervention group. All tests were twosided with a significance level of $P \leq 0.05$. Data were analyzed using SPSS version 24 (IBM Corporation, Armonk, NY, USA).
We applied the intention-to-treat principle in our analysis of intervention outcomes. To ensure that the results were as comprehensive as possible, we replaced missing data. The assumption that the SOC is stable over time guided our choice of imputation method. Therefore, we used the last observed carried forward (LOCF) method as our first choice. If the LOCF was not possible, we replaced missing values using the last observation carried backward (LOCB) method for each participant. If neither the LOCF nor the LOCB were possible, we replaced the missing values with the median score for the group.

\section{Results}

The results are based on a population of 131 participants who fulfilled the inclusion criteria and consented to participate (Figure 1).

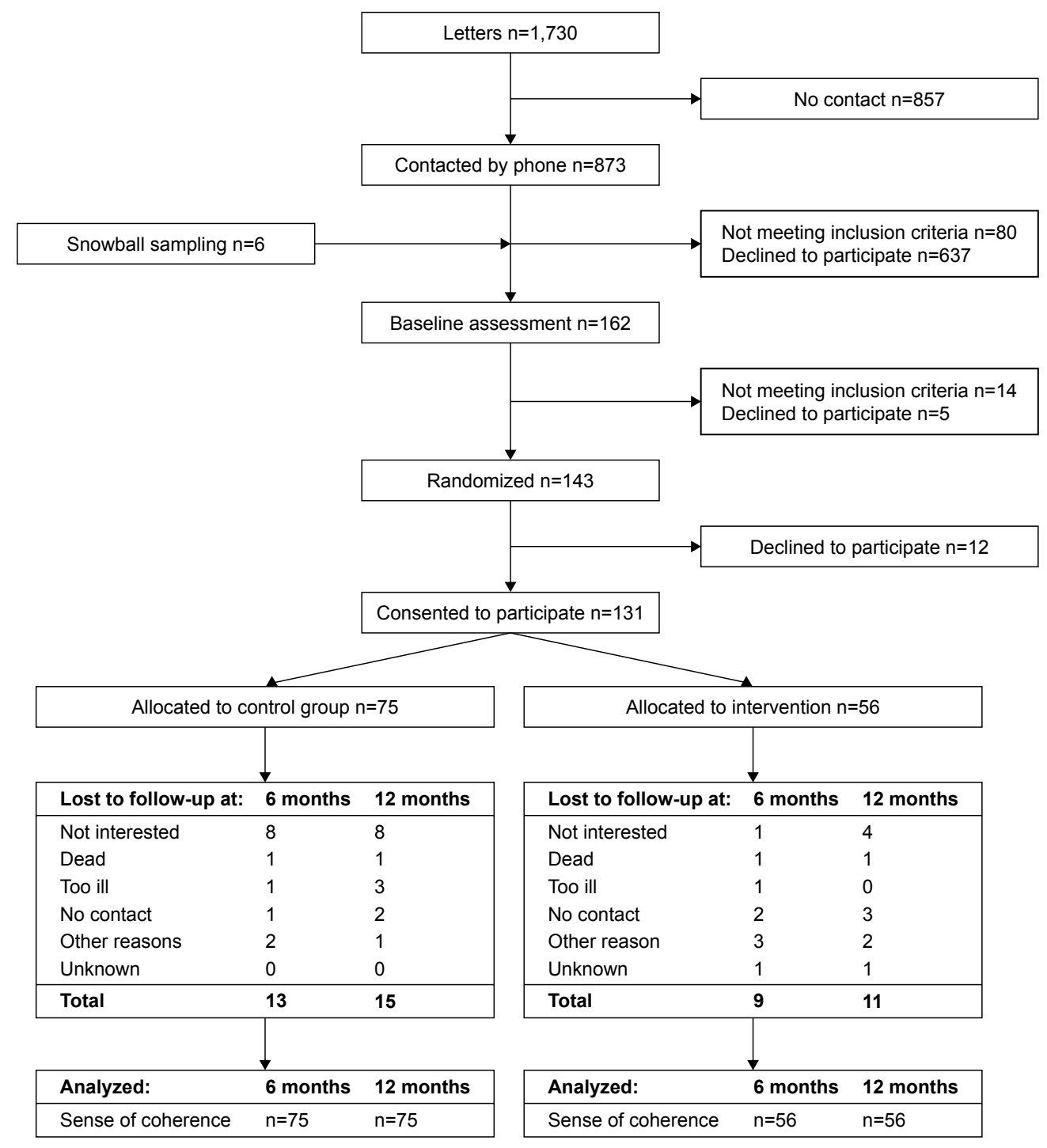

Figure I The flow of participants through the Promoting Aging Migrants' Capabilities study and the reasons for declining participation at 6-month and I2-month follow-up assessments. 
Table 2 Differences in the proportion of dropouts and nondropouts at 6 months and 12 months

\begin{tabular}{|c|c|c|c|c|c|c|}
\hline & \multicolumn{2}{|l|}{6 months } & \multirow[t]{2}{*}{$P$-value } & \multicolumn{2}{|l|}{12 months } & \multirow[t]{2}{*}{$P$-value } \\
\hline & $\begin{array}{l}\text { Dropouts } \\
\mathrm{n}=\mathbf{2 2}(\%)\end{array}$ & $\begin{array}{l}\text { Participants } \\
\mathrm{n}=109(\%)\end{array}$ & & $\begin{array}{l}\text { Dropouts } \\
\mathrm{n}=\mathbf{2 6}(\%)\end{array}$ & $\begin{array}{l}\text { Participants } \\
\mathrm{n}=105 \text { (\%) }\end{array}$ & \\
\hline Sex (male) & 73 & 46 & $0.022 *$ & 65 & 47 & 0.087 \\
\hline Living alone & 27 & 52 & $0.032 *$ & 35 & 51 & 0.124 \\
\hline Migrated from the Balkan Peninsula & 82 & 39 & $0.000 * * *$ & 81 & 37 & $0.000 * * *$ \\
\hline $\begin{array}{l}\text { Good self-rated overall ability to speak } \\
\text { Swedish when in contact with authorities }\end{array}$ & 68 & 81 & 0.190 & 62 & 83 & $0.018 *$ \\
\hline Mini-Mental State Examination $\geq 25$ points & 77 & 96 & $0.007 * *$ & 77 & 97 & $0.002 * *$ \\
\hline
\end{tabular}

Note: $* p<0.05, * * p<0.01, * * * p<0.001$.

We found no statistically significant differences between the control group and the intervention group in terms of demographic characteristics at baseline (see Table 1).

At the 6-month follow-up, 22 participants (17\%) had dropped out, and at the 12-month follow-up, 26 participants $(20 \%)$ had dropped out. There were no significant differences between the control group and the intervention group in terms of dropout rates. There was a significantly higher proportion of dropouts among males (6-month follow-up) and participants born in the Balkan Peninsula (6-month and 12-month follow-ups). The MMSE scores for dropouts were lower than those for nondropouts at both the 6-month and 12-month time points. Dropouts were also more likely to live alone (at 6 months) and evaluate their language skills as good (at 12 months). The most common cause for dropout was decreased interest in participation. Detailed information about dropout characteristics is given in Table 2.

\section{SOC}

Table 3 shows a cross section of the distribution of total SOC scores and the three components of SOC at baseline. There were no significant differences in SOC scores between the groups at baseline.

Table 4 reports changes in total SOC scores and SOC components at 6-month and 12-month postintervention. At the 6-month follow-up, we observed a significant difference in total SOC score in favor of the intervention group, $P=0.038$ (OR: 2.23; CI: $1.05-4.77$ ). There was no significant difference regarding the three components of SOC. However, when comparing the control group and intervention group in terms of the three components of SOC, participants in the intervention group were more likely to have maintained or strengthened scores for comprehensibility (OR: 1.49; CI: 0.72-3.07), manageability (OR: 1.96; CI: 0.94-4.11), and meaningfulness (OR: 1.50; CI: 0.69-3.25) at the 6-month follow-up. At 12 months, this difference did not maintain,

Table 3 Distribution of SOC scores and the three SOC components at baseline: mean (SD), median, n (\%)

\begin{tabular}{|c|c|c|c|c|}
\hline & $\begin{array}{l}\text { Control } \\
n=75\end{array}$ & $\begin{array}{l}\text { Intervention } \\
\mathrm{n}=56\end{array}$ & $P$-value & $\begin{array}{l}\text { Total } \\
n=|3|\end{array}$ \\
\hline \multicolumn{5}{|c|}{ SOC total score } \\
\hline Mean (SD) & 71.5 (I2.7) & $68.9(11.0)$ & 0.255 & $70.4(12.0)$ \\
\hline Median & 74.5 & 70.0 & & 73.0 \\
\hline n (\%) & $62(83)$ & $42(75)$ & & $104(74)$ \\
\hline \multicolumn{5}{|l|}{ Meaningfulness } \\
\hline Mean (SD) & $22.3(4.2)$ & $21.3(3.8)$ & 0.207 & $21.9(4.1)$ \\
\hline Median & 22.0 & 22.0 & & 22.0 \\
\hline n (\%) & $65(87)$ & $43(77)$ & & $108(82)$ \\
\hline \multicolumn{5}{|c|}{ Comprehensibility } \\
\hline Mean (SD) & $27.3(6.0)$ & $26.7(5.0)$ & 0.397 & $27.2(5.6)$ \\
\hline Median & 29.0 & 27.5 & & 28.0 \\
\hline $\mathrm{n}(\%)$ & $63(84)$ & $44(79)$ & & $107(82)$ \\
\hline \multicolumn{5}{|l|}{ Manageability } \\
\hline Mean (SD) & $21.8(2.8)$ & $20.7(3.2)$ & 0.210 & $21.3(4.5)$ \\
\hline Median & 23.0 & 22.0 & & 22.0 \\
\hline $\mathrm{n}(\%)$ & $64(85)$ & 44 (79) & & $108(82)$ \\
\hline
\end{tabular}

Abbreviation: SOC, sense of coherence. 
Table 4 Maintenance or improvement of total SOC scores and the scores of the three components at 6-month and 12-month follow-ups

\begin{tabular}{c|l|l|l}
\hline & $\begin{array}{l}\text { Control, } \\
\mathbf{n}(\%) \text { OR }\end{array}$ & $\begin{array}{l}\text { Intervention, } \mathbf{n}(\%) \\
\text { OR (95\% CI) }\end{array}$ & P-value \\
\hline 6 months & & & \\
\hline Total SOC & $43(57)$ & $42(75)$ & $0.038^{*}$ \\
& 1.00 & $2.23(1.05-4.77)$ & \\
\hline Meaningfulness & $50(67)$ & $42(75)$ & 0.303 \\
& 1.00 & $1.50(0.69-3.25)$ & \\
\hline Comprehensibility & $44(59)$ & $38(68)$ & 0.283 \\
& 1.00 & $1.49(0.72-3.07)$ & \\
\hline Manageability & $42(56)$ & $40(71)$ & 0.073 \\
& 1.00 & $1.96(0.94-4.11)$ & \\
\hline I2 months & & & \\
\hline Total SOC & $47(63)$ & $32(57)$ & 0.523 \\
& 1.00 & $0.79(0.39-1.61)$ & \\
\hline Meaningfulness & $49(65)$ & $37(66)$ & 0.930 \\
& 1.00 & $1.03(0.50-2.14)$ & \\
\hline Comprehensibility & $45(60)$ & $28(50)$ & 0.255 \\
& 1.00 & $0.67(0.33-1.34)$ & \\
\hline Manageability & $47(63)$ & $32(57)$ & 0.523 \\
& 1.00 & $0.79(0.39-1.61)$ & \\
\hline Notes) & &
\end{tabular}

Notes: $\mathrm{n}(\%), \mathrm{OR}, \mathrm{Cl}$, and $P$-values. $* P<0.05$.

Abbreviation: $\mathrm{SOC}$, sense of coherence. and the odds seemed to favor the control group regarding the maintenance of SOC.

Table 5 gives a longitudinal analysis of change between different levels of SOC in the control and intervention groups. Compared with the control group, more participants in the intervention group exhibited an increase in SOC level from the baseline to the 6-month follow-up (OR: 1.74; CI: 0.75-4.05), although the finding was not significant. At 12 months, SOC levels returned to baseline levels in the intervention group (Table 5). However, when comparing the level of SOC between the baseline and 12-month assessments, participants in the intervention group were more likely to have an increased SOC 12-month postintervention (OR: 1.14; CI: 0.45-2.87). This pattern of change was not observed in the control group.

\section{Discussion}

The first aim of this study was to evaluate the distribution of SOC, SOC ${ }^{12}$ among older persons who had undergone migration. The mean SOC score for the total group was $\approx 70$. Interestingly, the obtained scores were comparable with those of older native-born persons in the same age group.

Table 5 Changes in SOC levels between the control and intervention groups from baseline to 6-month and I2-month follow-ups

\begin{tabular}{|c|c|c|c|}
\hline & $\begin{array}{l}\text { Control, n=75 } \\
\text { n (\%) OR }\end{array}$ & $\begin{array}{l}\text { Intervention, n=56 } \\
n(\%) \text { OR }(95 \% \mathrm{Cl})\end{array}$ & $P$-value \\
\hline \multicolumn{4}{|c|}{ SOC level baseline to 6 months } \\
\hline Strengthened SOC & $\begin{array}{l}13(17) \\
1.00\end{array}$ & $\begin{array}{l}15(27) \\
1.74(0.75-4.05)\end{array}$ & 0.195 \\
\hline Maintained SOC & $\begin{array}{l}48(64) \\
1.00\end{array}$ & $\begin{array}{l}3 \mathrm{I}(55) \\
0.70(0.34-\mid .4 I)\end{array}$ & 0.318 \\
\hline Deteriorated SOC & $\begin{array}{l}14(19) \\
1.00\end{array}$ & $\begin{array}{l}10(18) \\
0.95(0.39-2.32)\end{array}$ & 0.906 \\
\hline \multicolumn{4}{|c|}{ SOC level 6 months to 12 months } \\
\hline Strengthened SOC & $\begin{array}{l}13(17) \\
1.00\end{array}$ & $\begin{array}{l}4(79) \\
0.37(0.11-1.19)\end{array}$ & 0.096 \\
\hline Maintained SOC & $\begin{array}{l}49(65) \\
1.00\end{array}$ & $\begin{array}{l}39(70) \\
1.22(0.58-2.56)\end{array}$ & 0.604 \\
\hline Deteriorated SOC & $\begin{array}{l}13(17) \\
1.00\end{array}$ & $\begin{array}{l}\text { I3 (23) } \\
\text { I.44 (0.6I-3.4I) }\end{array}$ & 0.405 \\
\hline \multicolumn{4}{|c|}{ SOC level baseline to 12 months } \\
\hline Strengthened SOC & $\begin{array}{l}12(16) \\
1.00\end{array}$ & $\begin{array}{l}10(18) \\
1.14(0.45-2.87)\end{array}$ & 0.779 \\
\hline Maintained SOC & $\begin{array}{l}50(67) \\
1.00\end{array}$ & $\begin{array}{l}31(55) \\
0.62(0.30-1.26)\end{array}$ & 0.189 \\
\hline Deteriorated SOC & $\begin{array}{l}13(17) \\
1.00\end{array}$ & $\begin{array}{l}15(27) \\
1.74(0.75-4.05)\end{array}$ & 0.195 \\
\hline
\end{tabular}

Abbreviation: SOC, sense of coherence. 
Specifically, the mean SOC score among native-born persons has been reported to range from 73 to $75 .{ }^{26}$ The difference is on score level, and it does not interfere with the levels of SOC, when the scores are divided into low, medium, and high level of SOC. When comparing the mean scores for different SOC components between older immigrants and older native-born persons, the same pattern can be seen: our participants scored 27 for comprehensibility (native born 27-28), 27 for manageability (native born 27-28), and 22 for meaningfulness (native born 23-24).

Previous research has described migration as a stressor that has a negative impact on SOC, leading older persons who have undergone migration to have a weaker SOC compared with native-born persons. ${ }^{27}$ However, our data indicate that the examined group of older participants had a SOC level that was comparable with that of native-born older persons. This provides a different picture of older persons who have undergone migration. One explanation for this could be that the participants in this study had been living in their host country for decades. During this time, they may have been able to create an understanding of the new context, the culture, and the society in the host country. This could make it easier to experience meaning in everyday life and to make everyday life more comprehensible and manageable. According to Antonovsky and Elfstadius, ${ }^{12}$ the meaningfulness element of SOC is the component that gives life emotional meaning. When a person experiences meaning in everyday life, it gives them motivation to act and to direct energy toward the challenges faced in everyday life.

Another explanation could be that persons willing to participate in health promotion initiatives, such as the one in this study, already have a stable and moderate SOC. This may be the consequence of stronger general resistance resources, such as social support and connections to one's own background and culture. ${ }^{12}$ This is in line with previous research, which indicates that social support derived from compatriots is important in supporting the experience of health in daily life..$^{8,25}$

The second aim of this study was to evaluate the longitudinal effects of health promotion intervention on SOC. Our results indicate that it might be possible to influence SOC via planned and focused health promotion activities. Table 4 shows a significant difference in total SOC score between the control and intervention groups at 6-month postintervention. The three components of SOC tended also to be in favor of intervention group, although the differences between the groups were not significant. The manageability component of SOC relates to the resources that a person possesses, which can be used to meet challenges in daily life. A strengthened sense of manageability has a concrete relevance for wellbeing because it corresponds to the perception that one has the resources needed to manage daily life. Is it likely that the person-centered frame of reference used in this intervention program enhanced manageability by providing participants with new ideas and strategies for dealing with challenges in daily life, including those caused by the aging process. According to Antonovsky and Elfstadius, ${ }^{12}$ a high sense of manageability is reflected in the perception that a person has agency. Our person-centered intervention used a peer learning approach based on the experiences and situations that the participants reported in daily life. Interviews have shown that when participants and professionals jointly raise awareness by acknowledging participants as capable persons and by confirming their abilities to act, ${ }^{33}$ participants experience an increased sense of being in charge of their daily life. This is also in line with the thoughts of Antonovsky and Elfstadius ${ }^{12}$ about the aim of health promotion. They argued that programs should be shaped such that encounters within the program result in the feeling that life can be predictable, that there is a balance, and that everyday life is meaningful.

Another aspect of the study that relates to possibilities for changing SOC is the movement between the different levels of SOC, both in positive and negative directions, as shown in Table 5. This can be related to the theory of salutogenesis, which holds that SOC is generally stable, such that while minor movements occur, SOC returns to one's individual, mean level. This pattern could be seen among participants in the intervention group but was not especially visible among participants in the control group. This indicates that while such health promotion interventions may temporarily shift SOC, more regular interventions might be necessary to strengthen SOC in a long-lasting way.

The aging process, which is accompanied by many physical changes, is generally thought of as a stressor that can create the feeling that everyday life is difficult to manage. This can in turn affect one's sense of meaning in daily life. However, regular person-centered health promotion interventions might be able to make aging more comprehensible and manageable. Such interventions might also create opportunities for encounters with other persons with similar experiences, and thus enable peer support in daily life. The person-centered approach is focused on creating opportunities for the person to prioritize health and make decisions that support health and well-being without focusing solely on the physical signs of frailty or declined capacity. ${ }^{31}$ 


\section{Methodological considerations}

We used the 13-item version of the Orientation to Life Questionnaire, ${ }^{12}$ ie, the SOC-13 scale, to assess SOC. The validity and reliability of the SOC-13 have been confirmed and the scale seems to be able to assess life orientation in a reliable and valid way. The instrument is also applicable in different cultural contexts. ${ }^{36}$ However, the SOC-13 is composed of three separate concepts in factor analysis. There might be a risk that this fact has created a familywise error rate, which might have an impact on the reliability. Therefore, the interpretation of the results of the three separate components should be considered as just an indication of how the intervention might have affected separate parts of the SOC.

One strength of this study is that we used a randomized controlled design to evaluate the effect of our health promotion intervention on SOC. RCTs in the field of health promotion targeting older persons aging in the context of migration are scares. ${ }^{38}$ One possible reason for this is the methodological challenges these studies have met, which were also evident in our study. Methodological challenges were encountered in the recruitment procedure. Despite conducting three recruitment waves, we failed to recruit 65 participants in each study arm required to reach power. In addition, there was a higher dropout among participants in the intervention group. This resulted in an unequal number of participants in the control and intervention groups. Thus, we failed to reach power, which is a methodological limitation. This may have affected the ability to detect significant differences between groups.

However, although we found a significant effect of the intervention on total SOC score at the 6-month follow-up, our analysis suggests that we might have found more significant differences between groups if we had been able to recruit a larger sample.

The dropout rate was $20 \%$ at 1 -year follow-up, which is in line with another study targeting older persons. ${ }^{39}$ There is no specific level of loss to follow-up at which dropouts becomes a problem. However, a dropout rate at the level of $20 \%$ has to be considered as a possible bias ${ }^{40}$ Studies including older persons have a generally higher loss to follow-up than studies with younger participants, which is a challenge in research on aging. ${ }^{41}$ The small sample, in combination with the number of dropouts, may have affected the internal validity of the results.

The main reason for dropout was that participants were no longer interested in taking part in the is study, which had an impact on the adherence rate. The lack of interest indicates that the content of the intervention may not have been directly related to the everyday lives of certain participants. If the older persons in our study had better health than we had expected, other themes more closely associated with the needs of healthy older persons might have been perceived to be more interesting. This may be seen as a limitation, but may also be considered an important contribution to intervention research targeting older people aging in the context of migration.

Another reason might be related to the inclusion criteria, and especially the age range, which was set to 70 years or older based on recommendations in previous research. ${ }^{34}$ This age might have been too low, as many individuals in this age group are able to easily manage daily life without any support from health care services. These interpretations of reasons for dropout may be supported by results from studies with native-born persons. ${ }^{39,42}$ In those studies, the age of participants was higher. Additionally, the dropout rate was higher in the control group than in the intervention group, indicating that participants in the intervention group found the content and participation more useful than did the participants in our study. Further, some individuals may have declined to participate due to a distrust of health care providers, which has been explored in qualitative studies describing older immigrants' health perceptions. ${ }^{43-45}$ Still, the OR calculations can be interpreted to support the hypothesis that the intervention had an impact on SOC, even if the effect did not last.

The analyses were based on the intention-to-treat principle. To obtain comprehensive results, we replaced missing SOC data with LOCF or LOCB for each individual dropout. We chose this replacement method based on theoretical assumptions connected to the SOC. Specifically, the SOC is thought to be quite stable in old age and is an individual measure. We are, therefore, confident that our method for replacing missing data was appropriate.

The intervention and control groups had similar sociodemographic characteristics, indicating that the external validity of the study was satisfactory. However, our results should be interpreted with caution because of the lack of statistically significant between-group differences. Indeed, more research is needed with larger participant populations. Further research targeting older persons with immigrant backgrounds could also examine why this population might decline to participate in research projects.

\section{Conclusion}

The results of this study indicate that older persons who have undergone migration do not differ from older native-born persons in terms of SOC in everyday life. Our data imply 
that it might be possible to support SOC by health promotion interventions with a person-centered approach.

Our study population had been living in their host country for decades, and they had settled down permanently. That they have lived a long time in the host country indicates that they had undergone acculturalization, and so at this point in life, the experience of immigration was not negatively impacting their SOC, instead, there might be other factors which affects health in daily life. Therefore, it is appropriate to describe this group of older persons as persons with their own narratives and specific life events, which has formed their SOC. Our results can be a first step to create a more nuanced image of older immigrants. Indeed, members of this population may have overcome past challenges and do not appear to differ from the majority of similarly aged older persons in terms of health problems. This could indicate that this population does not have any specific needs regarding health, aside from needs shared with other people of the same age. Thus, the structure of health promotion interventions for older persons with an immigrant background could be similar to those for native-born persons. The most important issue is that the health promotion has a person-centered approach. This will ensure that interventions begin by considering each individual person as a person and not as an immigrant. While migration background may be an important factor in some challenges in daily life, the specific narrative of an individual, and not their cultural or ethnic background, will likely be more helpful in allocating appropriate resources to individuals.

\section{Ethics approval}

The regional ethical review board in Gothenburg, Sweden, granted formal ethical approval (Reg nr: 821-11). Regional Ethical Review Board, PO Box 401, SE405 30 Gothenburg. https://www.epn.se/goeteborg/om-naemnden/.

\section{Acknowledgments}

This work was financially supported by Hjalmar Svensson Foundation and the Centre for Person-Centred Care, GPCC, University of Gothenburg, Sweden. GPCC is funded by the Swedish Government's grant for Strategic Research Areas, Care Sciences (Application to Swedish Research Council nr 2009-1088). The funders played no role in the execution or writing of the manuscript. This study was accomplished, while first author was affiliated with the Swedish National Graduate School for Competitive Science on Ageing and Health (SWEAH), which is funded by the Swedish Research Council. The research was accomplished at the Sahlgrenska Academy, University of Gothenburg, Sweden.

\section{Disclosure}

The authors report no conflicts of interest in this work.

\section{References}

1. White P. Migrant populations approaching old age: prospects in Europe. J Ethn Migr Stud. 2006;32(8):1283-1300.

2. United Nations, Department of Economic and Social Affairs, Population Division. World Population Prospects: The 2015 Revision, Key Findings and Advance Tables. 2015. Working Paper No. ESA/P/ WP.241.

3. UNFPA. Ageing in the Twenty-First Century: A Celebration and a Challenge. New York: United Nations Population Fund: 2012.

4. Gustafsson C. Äldre invandrares levnadsförhållanden i Sverige: En bakgrund inför framtida forskning [Elderly immigrants' living conditions in Sweden: A background for future research]. Umeå: Umeå University; 2016.

5. SCB [webpage on the Internet]. SCB. statistikdatabasen $>$ Befolknings statistik > Total folkmängd. 2016. Available from: http://www.statistikdatabasen.scb.se/pxweb/sv/ssd/START_BE_BE0101_BE0101E/ FodelselandArK/?rxid=af45d1c8-19d5-45e4-ab72-01 ccfba9fb7f. Accessed September 19, 2018.

6. Torres S. Elderly Immigrants in Sweden: 'Otherness' Under Construction. J Ethn Migr Stud. 2006;32(8):1341-1358.

7. Karl U, Torres S. Ageing in Contexts of Migration. Abingdon, Oxon/ New York, NY: Routledge; 2016.

8. Diaz E, Kumar BN. Differential utilization of primary health care services among older immigrants and Norwegians: a register-based comparative study in Norway. BMC Health Serv Res. 2014;14:623.

9. Hjern A. Migration and public health: Health in Sweden: The National Public Health Report 2012. Chapter 13. Scand J Public Health. 2012; 40(9 Suppl):255-267.

10. Kulla G, Sarvimäki A, Fagerström L. Health resources and health strategies among older Swedish-speaking Finns - a hermeneutic study. Scand J Caring Sci. 2006;20(1):51-57.

11. Johansson K, Rudman DL, Mondaca M, et al. Moving Beyond 'Aging In Place' to Understand Migration and Aging: Place Making and the Centrality Of Occupation. J Occup Sci. 2013;20(2):108-119.

12. Antonovsky A, Elfstadius M. Hälsans mysterium [Unravelling the mystery of health]. Stockholm: Natur och kultur; 2005.

13. Ebrahimi Z, Wilhelmson K, Eklund K, Moore CD, Jakobsson A. Health despite frailty: exploring influences on frail older adults' experiences of health. Geriatr Nurs. 2013;34(4):289-294.

14. Lood Q. Discovering the capabilities of ageing persons who are born abroad: crossing norms, moving health promotion forward. Göteborg: Department of Clinical Neuroscience and Rehabilitation, Institute of Neuroscience and Physiology, Section for Health and Rehabilitation Sahlgrenska Academy at University of Gothenburg; 2015.

15. Hasselkus BR. The Meaning of Everyday Occupation. 2nd ed. Thorofare, NJ: SLACK; 2011.

16. Slootjes J, Keuzenkamp S, Saharso S. The mechanisms behind the formation of a strong Sense of Coherence (SOC): The role of migration and integration. Scand J Psychol. 2017;58(6):571-580.

17. Gupta J, Sullivan C. The Central Role of Occupation in the Doing, Being and Belonging of Immigrant Women. J Occup Sci. 2013;20(1): 23-35.

18. Wright-St Clair VA, Nayar S. Older Asian immigrants' participation as cultural enfranchisement. J Occup Sci. 2016:64-75.

19. Szczepura A. Access to health care for ethnic minority populations. Postgrad Med J. 2005;81(953):141-147.

20. Eriksson M. Salutogenes: om hälsans ursprung: från forskning till praktisk tillämpning. [Salutogenesis: The origin of health: from research to practical implementation] Stockholm: Liber; 2015.

21. Kulla G, Ekman S-L, Sarvimäki A. Experiential health from an ageing and migration perspective: the case of older Finland-Swedes. J Immigr Minor Health. 2010;12(1):93-99. 
22. Hocking $\mathrm{C}$. Contribution of occupation to health and well-being. In: Boyt Schell BA, Gillen G, Scaffa ME, Cohn ES, editors. Willard and Spackman's Occupational Therapy. 12th ed. Philadelphia: Wolters Kluwer Health/Lippincott Williams \& Wilkins Corp; 2013:72-81.

23. Wilcock AA, Hocking C. An Occupational Perspective of Health. Thorofare, NJ: Slack; 2015.

24. Ekman I, Norberg A, Swedberg K. Tillämning av personcentrering inom hälso-och sjukvård. In: Ekman I, editor. Personcentrering inom hälso-och sjukvård. Stockholm: Liber AB; 2014:69-86.

25. Eriksson M, Lindström B, Lilja J. A sense of coherence and health. Salutogenesis in a societal context: Åland, a special case? J Epidemiol Community Health. 2007;61(8):684-688.

26. Lindmark U, Stenström U, Gerdin EW, Hugoson A. The distribution of "sense of coherence" among Swedish adults: a quantitative cross-sectional population study. Scand J Public Health. 2010;38(1): $1-8$.

27. Erim Y, Morawa E, Atay H, Aygn S, Gkalp P, Senf W. Sense of coherence and depression in the framework of immigration: Turkish patients in Germany and in Turkey. Int Rev Psychiatry. 2011; 23(66):542-549.

28. Hulter Åsberg K. ADL-trappan. [ADL-Staircase] Lund: Studentlitteratur. 1990.

29. Folstein MF, Folstein SE, McHugh PR. "Mini-mental state". A practical method for grading the cognitive state of patients for the clinician. $J$ Psychiatr Res. 1975;12(3):189-198.

30. Sadler GR, Lee HC, Lim RS, Fullerton J. Recruitment of hard-to-reach population subgroups via adaptations of the snowball sampling strategy. Nurs Health Sci. 2010;12(3):369-374.

31. Fors A, Ekman I. Personcentrerade samtal inom hälsopromotivt arbete. In: Ekman I, editor. Personcentrering inom hälso-och sjukvård: Från filosofi till praktik. [Person-centered healthcare: From philosophy to practice] Stockholm: Liber, Stockholm; 2014:226-244.

32. Barenfeld E. How to Support Knowing and Doing in Promotion of Health: Lessons Learned From the Promoting Aging Migrants' Capabilities Program. Göteborgs universitet: Göteborg; 2016.

33. Barenfeld E, Gustafsson S, Wallin L, Dahlin-Ivanoff S. Understanding the "black box" of a health-promotion program: Keys to enable health among older persons aging in the context of migration. Int J Qual Stud Health Well-being. 2015;10:29013.

34. Gustafsson S, Lood Q, Wilhelmson K, Häggblom-Kronlöf G, Landahl S, Dahlin-Ivanoff S. A person-centred approach to health promotion for persons 70+ who have migrated to Sweden: promoting aging migrants' capabilities implementation and RCT study protocol. BMC Geriatr. 2015;15:10
35. Lood Q, Gustafsson S, Dahlin Ivanoff S. Bridging barriers to health promotion: a feasibility pilot study of the 'Promoting Aging Migrants' Capabilities study'. J Eval Clin Pract. 2015;21(4):604-613.

36. Eriksson M, Lindström B. Validity of Antonovsky's sense of coherence scale: a systematic review. J Epidemiol Community Health. 2005; 59(6):460-466.

37. Dahlin-Ivanoff S, Gosman-Hedström G, Edberg AK, et al. Elderly persons in the risk zone. Design of a multidimensional, health-promoting, randomised three-armed controlled trial for "prefrail" people of 80+ years living at home. BMC Geriatr. 2010;10:27.

38. Lood Q, Häggblom-Kronlöf G, Dahlin-Ivanoff S. Health promotion programme design and efficacy in relation to ageing persons with culturally and linguistically diverse backgrounds: a systematic literature review and meta-analysis. BMC Health Serv Res. 2015;15:560.

39. Behm L, Wilhelmson K, Falk K, Eklund K, Zidén L, Dahlin-Ivanoff S. Positive health outcomes following health-promoting and diseasepreventive interventions for independent very old persons: long-term results of the three-armed RCT Elderly Persons in the Risk Zone. Arch Gerontol Geriatr. 2014;58(3):376-383.

40. Dumville JC, Torgerson DJ, Hewitt CE. Reporting attrition in randomised controlled trials. BMJ. 2006;332(7547):969-971.

41. Hardy SE, Allore H, Studenski SA. Missing data: a special challenge in aging research. J Am Geriatr Soc. 2009;57(4):722-729.

42. Behm L, Eklund K, Wilhelmson K, et al. Health promotion can postpone frailty: results from the RCT elderly persons in the risk zone. Public Health Nurs. 2016;33(4):303-315.

43. Barenfeld E, Wallin L, Björk Brämberg E. Moving from knowledge to action in partnership: A case study on program adaptation to support optimal aging in the context of migration. J Appl Gerontol. Epub 2017 Aug 1.

44. Lood Q, Häggblom-Kronlöf G, Dellenborg L. Embraced by the past, hopeful for the future: meaning of health to ageing persons who have migrated from the Western Balkan region to Sweden. Ageing Soc. 2016; 36(3):649-665.

45. Arola LA, Mårtensson L, Häggblom Kronlöf G. Viewing oneself as a capable person - experiences of professionals working with older Finnish immigrants. Scand J Caring Sci. 2017;31(4):759-767.
Clinical Interventions in Aging

\section{Publish your work in this journal}

Clinical Interventions in Aging is an international, peer-reviewed journal focusing on evidence-based reports on the value or lack thereof of treatments intended to prevent or delay the onset of maladaptive correlates of aging in human beings. This journal is indexed on PubMed Central, MedLine,

\section{Dovepress}

CAS, Scopus and the Elsevier Bibliographic databases. The manuscript management system is completely online and includes a very quick and fair peer-review system, which is all easy to use. Visit http://www.dovepress. com/testimonials.php to read real quotes from published authors. 